\title{
Tensor models and 3-ary algebras
}

Naoki Sasakura, a)

Yukawa Institute for Theoretical Physics, Kyoto University, Kyoto 606-8502, Japan

(Dated: 13 November 2018)

Tensor models are the generalization of matrix models, and are studied as models of quantum gravity in general dimensions. In this paper, I discuss the algebraic structure in the fuzzy space interpretation of the tensor models which have a tensor with three indices as its only dynamical variable. The algebraic structure is studied mainly from the perspective of 3-ary algebras. It is shown that the tensor models have algebraic expressions, and that their symmetries are represented by 3 -ary algebras. It is also shown that the 3-ary algebras of coordinates, which appear in the nonassociative fuzzy flat spacetimes corresponding to a certain class of configurations with Gaussian functions in the tensor models, form Lie triple systems, and the associated Lie algebras are shown to agree with those of the Snyder's noncommutative spacetimes. The Poincare transformations of the coordinates on the fuzzy flat spacetimes are shown to be generated by 3-ary algebras.

PACS numbers: 04.60.-m, 04.60.Pp, 02.40.Gh

Keywords: Tensor models, 3-ary algebras, Quantum gravity, Fuzzy spaces

a) Electronic mail: sasakura@yukawa.kyoto-u.ac.jp 


\section{INTRODUCTION}

Tensor models have originally been introduced $\underline{1-3}$ to describe the simplicial quantum gravity in more than two dimensions, with the hope to extend the successful description of the two-dimensional simplicial quantum gravity by the matrix models to other dimensions. The formulation has later been extended $\underline{4}^{-\underline{6}}$ to describe spin foam and loop quantum gravities by considering Lie-group valued indices ${ }^{7}$. Despite various difficulties ${ }^{8}$ and the rather slow development since the introduction of tensor models, some interesting results have been reported recently $\underline{\underline{9}-15} \underline{\underline{15}}$. These developments strengthen the general belief that tensor models indicate the right direction to the background independent formulation of quantum gravity.

Concerning the background independence of quantum gravity, the dimensions of spacetime should be regarded as an effective dynamical quantity rather than a given constant. This viewpoint not only seems natural from the physical requirement of quantum gravity, but has also been supported by some recent results from simplicial quantum gravity $\underline{16}$ and field theoretical treatment $\frac{17}{}$. On the other hand, however, the original formulation of tensor models as well as the group field theory depend on the considering dimensions in their formalism through the rank of tensors and the choices of groups. Therefore it would be desired to find another interpretation of tensor models which singles out a tensor model that is applicable to general dimensions.

The simplest choice of a tensor model is to consider one which has a tensor with three indices as its only dynamical variable. Then, by identifying the rank-three tensor with the structure constant of an algebra charactering a fuzzy space, the tensor model can be interpreted as theory of a dynamical fuzzy space. Since one can in principle choose the values of the rank-three tensor to construct fuzzy spaces corresponding to any dimensional spaces, the rank-three tensor models can equally treat spaces in general dimensions. This idea has first been presented in Ref. 18, and the subsequent studies mainly in numerical methods have supported the validity of this basic idea $\underline{19} \underline{26}$. The purpose of the present paper is to provide a full treatment of the original incomplete presentation of the idea, and to pursue the algebraic description of the tensor models. In the sequel, it is found that 3-ary algebras $27-29$ describe the symmetries of the tensor models. 3-ary algebras have been

introduced in physics by $\mathrm{Nambu}^{30}$, and have recently been widely discussed in the context of M-theory ${ }^{31} \underline{33}$. This unexpected common appearance of 3-ary algebras suggests the general 
importance of this new way of describing symmetry in the physics of quantum spacetime.

This paper is organized as follows. In the following section, the rank-three tensor model is presented. In Section III, the structure of the algebras corresponding to the rank-three tensor models is discussed. In Section IV, the commutative case of the algebras is discussed. In Section V, the rank-three tensor model is described in terms of the algebras. In Section VI, the fuzzy flat spacetimes with the algebras corresponding to a certain class of configurations with Gaussian functions in the rank-three tensor models are discussed. In Section VII, the 3-ary algebra of the coordinates obtained in the previous section is studied, and a connection with the Snyder's noncommutative spacetime is found. In Section VIII, the Poincare symmetry of the fuzzy flat spacetimes is shown to be represented by 3-ary operations. The final section is devoted to summary, discussions and future prospects.

\section{THE RANK-THREE TENSOR MODELS}

The simplest generalization of the matrix models will be the tensor models which have a tensor with three indices $M_{a b c}(a, b, c=1,2, \ldots, N)$ as their only dynamical variable $\mathrm{e}^{\underline{\underline{1}}-\underline{3}}$. The $M_{a b c}$ takes complex values, and the hermiticity of a matrix is generalized to

$$
M_{a b c}=M_{b c a}=M_{c a b}=M_{b a c}^{*}=M_{a c b}^{*}=M_{c b a}^{*}
$$

which states that the tensor takes its complex conjugate under odd permutations of the indices, while they are unchanged under even permutations.

Because of the property (1), the symmetry which can be associated to the tensor models is the real orthogonal group symmetry,

$$
M_{a b c} \rightarrow O_{a}{ }^{a^{\prime}} O_{b}{ }^{b^{\prime}} O_{c}{ }^{c^{\prime}} M_{a^{\prime} b^{\prime} c^{\prime}}, \quad O \in O(N, R)
$$

instead of the unitary groups for the hermitian matrix models.

Let me define a new tensor,

$$
C_{a b c}=M_{a b c}+M_{b a c}+i\left(M_{a b c}-M_{b a c}\right) .
$$

Because of the generalized hermiticity condition (11), this tensor is real, and is invariant under the cyclic permutations of the indices,

$$
C_{a b c}=C_{b c a}=C_{c a b}
$$


Note that $C_{a b c}$ has no dependence under the odd permutations of the indices. The symmetry transformation for $C_{a b c}$ is given by a formula similar to (2).

In fact, $C_{a b c}$ is equivalent to $M_{a b c}$ as degrees of freedom, because, from (3), one can show that $M_{a b c}$ can also be expressed by $C_{a b c}$ as

$$
M_{a b c}=\frac{1}{4}\left(C_{a b c}+C_{b a c}\right)-\frac{i}{4}\left(C_{a b c}-C_{b a c}\right) .
$$

Therefore, a tensor model with real $C_{a b c}$ satisfying (4) is equivalent to the one with complex $M_{a b c}$ satisfying (11). In the following discussions, real $C_{a b c}$ with (4) will be used.

\section{PROPERTIES OF CORRESPONDING ALGEBRAS}

In this section, I will discuss an interpretation of the tensor models in terms of the notion of fuzzy spaces.

A fuzzy space is characterized by an algebra of functions $\phi_{a}(a=1,2, \ldots, N)$ on it, which form a basis. Generally, the multiplication of such an algebra is defined by a structure constant $f_{a b}^{c}$ as

$$
\phi_{a} \phi_{b}=f_{a b}^{c} \phi_{c}
$$

where $f_{a b}{ }^{c}$ is assumed to be real to describe a real fuzzy space. It is important to note that the algebra may not be associative; rather, nonassociativity will play essential roles in the discussions of this paper ${ }^{34}$.

I also assume there exists a metric,

$$
\left\langle\phi_{a} \mid \phi_{b}\right\rangle=h_{a b}
$$

which is assumed to be real, symmetric, $h_{a b}=h_{b a}$, and bilinear.

The basis of functions $\phi_{a}$ can be changed to another one. A new basis may be given by functions of $\phi_{a}$. By using (6) , any nonlinear function of $\phi_{a}$ can be expressed linearly, because

$$
\begin{aligned}
\phi_{a}^{\prime} & =A_{a}^{b} \phi_{b}+A_{a}^{b c} \phi_{b} \phi_{c}+\cdots \\
& =\left(A_{a}{ }^{d}+A_{a}^{b c} f_{b c}{ }^{d}+\cdots\right) \phi_{d},
\end{aligned}
$$

where $A$ 's are real numerical coefficients. Therefore, any new basis provided by functions of $\phi_{a}$ can in fact be obtained by a linear transformation of $\phi_{a}$. Since the change of basis should be invertible, it is given by a general linear group transformation,

$$
\phi_{a} \rightarrow M_{a}^{a^{\prime}} \phi_{a^{\prime}}, \quad M \in G L(N, R)
$$


Tensor models and 3-ary algebras

Under this general linear group transformation, the $f_{a b}{ }^{c}$ and $h_{a b}$ are transformed by

$$
\begin{aligned}
f_{a b}{ }^{c} & \rightarrow M_{a}^{a^{\prime}} M_{b}^{b^{\prime}} M_{c^{\prime}}^{-1 c} f_{a^{\prime} b^{\prime}} c^{\prime}, \\
h_{a b} & \rightarrow M_{a}^{a^{\prime}} M_{b}^{b^{\prime}} h_{a^{\prime} b^{\prime}} .
\end{aligned}
$$

The above transformations of the basis functions may be interpreted as the fuzzy space analogue to the diffeomorphisms of an ordinary space, since a diffeomorphism generates a linear transformation of a basis of functions on it 18 .

To relate the above algebraic structure with the rank-three tensor models, let me assume a relation,

$$
C_{a b c}=f_{a b}{ }^{\prime} h_{c^{\prime} c}
$$

Then the property of the cyclic symmetry (44) can be translated to the following cyclic condition on the algebra as

$$
\left\langle\phi_{a} \phi_{b} \mid \phi_{c}\right\rangle=\left\langle\phi_{a} \mid \phi_{b} \phi_{c}\right\rangle=\left\langle\phi_{b} \mid \phi_{c} \phi_{a}\right\rangle
$$

The former equality is because

$$
\begin{aligned}
& \left\langle\phi_{a} \phi_{b} \mid \phi_{c}\right\rangle=f_{a b}^{d}\left\langle\phi_{d} \mid \phi_{c}\right\rangle=f_{a b}^{d} h_{d c}=C_{a b c}, \\
& \left\langle\phi_{a} \mid \phi_{b} \phi_{c}\right\rangle=f_{b c}{ }^{d}\left\langle\phi_{a} \mid \phi_{d}\right\rangle=f_{b c}{ }^{d} h_{a d}=C_{b c a} .
\end{aligned}
$$

The proof of the latter is similar.

Now let me define a 3 -ary product by ${ }^{35}$

$$
\left[\phi_{a}, \phi_{b} ; \phi_{c}\right] \equiv\left(\phi_{a} \phi_{c}\right) \phi_{b}-\left(\phi_{b} \phi_{c}\right) \phi_{a}
$$

The first two entries are antisymmetric,

$$
\left[\phi_{a}, \phi_{b} ; \phi_{c}\right]=-\left[\phi_{b}, \phi_{a} ; \phi_{c}\right]
$$

The metric is invariant under the 3-ary operation on the third entry,

$$
\left\langle\left[\phi_{a}, \phi_{b} ; \phi_{c}\right] \mid \phi_{d}\right\rangle=-\left\langle\phi_{c} \mid\left[\phi_{a}, \phi_{b} ; \phi_{d}\right]\right\rangle
$$

This can be proved by using (12) as

$$
\begin{aligned}
\left\langle\left[\phi_{a}, \phi_{b} ; \phi_{c}\right] \mid \phi_{d}\right\rangle & =\left\langle\left(\phi_{a} \phi_{c}\right) \phi_{b}-\left(\phi_{b} \phi_{c}\right) \phi_{a} \mid \phi_{d}\right\rangle \\
& =\left\langle\left(\phi_{a} \phi_{c}\right) \phi_{b} \mid \phi_{d}\right\rangle-\left\langle\left(\phi_{b} \phi_{c}\right) \phi_{a} \mid \phi_{d}\right\rangle \\
& =\left\langle\phi_{a} \phi_{c} \mid \phi_{b} \phi_{d}\right\rangle-\left\langle\phi_{b} \phi_{c} \mid \phi_{a} \phi_{d}\right\rangle \\
& =\left\langle\phi_{c} \mid\left(\phi_{b} \phi_{d}\right) \phi_{a}\right\rangle-\left\langle\phi_{c} \mid\left(\phi_{a} \phi_{d}\right) \phi_{b}\right\rangle \\
& =-\left\langle\phi_{c} \mid\left[\phi_{a}, \phi_{b} ; \phi_{d}\right]\right\rangle .
\end{aligned}
$$


This invariance can be discussed more explicitly by writing down the linear transformation associated to (14) as

$$
\begin{aligned}
{\left[\phi_{a}, \phi_{b} ; \phi_{c}\right] } & =\left(\phi_{a} \phi_{c}\right) \phi_{b}-\left(\phi_{b} \phi_{c}\right) \phi_{a} \\
& =f_{a c}{ }^{d} f_{d b}{ }^{e} \phi_{e}-f_{b c}{ }^{d} f_{d a}{ }^{e} \phi_{e} \\
& =\left(M_{a b}\right)_{c}{ }^{d} \phi_{d},
\end{aligned}
$$

where

$$
\left(M_{a b}\right)_{c}^{d}=f_{a c}^{e} f_{e b}{ }^{d}-f_{b c}{ }^{e} f_{e a}{ }^{d} .
$$

The indices of $\left(M_{a b}\right)_{c}{ }^{d}$ have the following anti-symmetric properties,

$$
\begin{aligned}
& \left(M_{a b}\right)_{c}{ }^{d}=-\left(M_{b a}\right)_{c}{ }^{d} \\
& \left(M_{a b}\right)_{c d}=-\left(M_{a b}\right)_{d c}
\end{aligned}
$$

where $\left(M_{a b}\right)_{c d}=\left(M_{a b}\right)_{c}{ }^{d^{\prime}} h_{d^{\prime} d}$. The latter equation can be proven by the relation (11) and the cyclic property (41).

Especially, the latter equation of (20) shows that $h_{a b}$ is invariant under the infinitesimal transformation (18). Therefore there exist various invariant quantities under the transformation. For example, if $h_{a b}$ is invertible,

$$
h^{a b} \phi_{a} \phi_{b}
$$

is invariant, where $h^{a b}$ is the inverse of $h_{a b}$. It is clear that one can construct various invariants by contracting the lower indices of $\phi_{a}$ with $h^{a b}$.

Another kind of invariant is the trace defined by

$$
\operatorname{Tr}(\mathcal{O}) \equiv h^{a b}\left\langle\phi_{a} \mid \mathcal{O} \phi_{b}\right\rangle=h^{a b}\left\langle\phi_{a} \mathcal{O} \mid \phi_{b}\right\rangle
$$

When $\mathcal{O}$ is an invariant, the whole expression $\operatorname{Tr}(\mathcal{O})$ is also invariant. The latter equation is valid, because of (12).

It should be stressed that, due to the general possibility of nonassociativity of the algebra, the 3-ary operation (18) on the third entry does not satisfy the Leibnitz rule in general ${ }^{36}$. Therefore, for example, the invariant (21) is not invariant under the 3-ary operation to the whole expression,

$$
\left[\phi_{a}, \phi_{b} ; h^{c d} \phi_{c} \phi_{d}\right] \neq 0 \text { in general }
$$


while the following equation,

$$
h^{c d}\left[\phi_{a}, \phi_{b} ; \phi_{c}\right] \phi_{d}+h^{c d} \phi_{c}\left[\phi_{a}, \phi_{b} ; \phi_{d}\right]=0
$$

holds.

The orthogonal group symmetry (2) of the tensor models and the general linear symmetry (9) of the algebra can be identified by partial gauge fixing of the latter symmetry as follows. Let me assume that the metric $h_{a b}$ is positive definite. Then the transformation (10) allows $h_{a b}$ to be gauge fixed to

$$
h_{a b}=\delta_{a b}
$$

Under the gauge fixing (25), the remaining symmetry agrees with the orthogonal group symmetry (2) of the tensor models. In this case, the Lie group generated by the infinitesimal transformation (18) is a subgroup of the orthogonal group $O(N, R)$ in general.

With the gauge fixing (25), the structure constant of the algebra and the dynamical variable of the tensor models can be identified by

$$
C_{a b c}=f_{a b}^{c} .
$$

Thus, the degrees of freedom of fuzzy spaces and those of the tensor models coincide.

\section{IMPOSING COMMUTATIVITY OF THE ALGEBRA}

In the following discussions, let me assume that the tensor $C_{a b c}$ is not only cyclic symmetric but totally symmetric,

$$
C_{a b c}=C_{b c a}=C_{c a b}=C_{b a c}=C_{a c b}=C_{c b a} .
$$

This reduction of the degrees of freedom has been introduced previously $\underline{20}$ to simplify the analysis of the tensor models. In fact, this reduction of the degrees of freedom does not diminish the physical interests in the tensor models, since various interesting properties in relation with the general relativity have been shown ${ }^{21}-2 \underline{\underline{2}}$ under the reduction.

In addition to the property (12), the total symmetry requires that the algebra be commutative,

$$
\phi_{a} \phi_{b}=\phi_{b} \phi_{a}
$$


While the commutators between $\phi_{a}$ vanish on account of the commutativity (28), the 3 -ary product (14) is equivalent to an associator ${ }^{37}$,

$$
\left[\phi_{a}, \phi_{b} ; \phi_{c}\right]=\left(\phi_{a} \phi_{c}\right) \phi_{b}-\phi_{a}\left(\phi_{c} \phi_{b}\right)
$$

and takes non-vanishing values in general, reflecting the nonassociativity of the algebra.

In the commutative case, the following cyclic identity holds,

$$
\left[\phi_{a}, \phi_{b} ; \phi_{c}\right]+\left[\phi_{b}, \phi_{c} ; \phi_{a}\right]+\left[\phi_{c}, \phi_{a} ; \phi_{b}\right]=0
$$

This can be shown from (28) and (29) .

As in the general non-commutative case discussed in the previous section, the associator (29) does not satisfy the Leibnitz rule in general also for the commutative case. However, there exists a physically interesting 3-Leibnitz subalgebra 28,29 which satisfies a kind of Leibnitz rule and is intimately related to the tensor models. This will be discussed in Section VII.

\section{ALGEBRAIC DESCRIPTION OF THE RANK-THREE TENSOR MODELS}

After the gauge fixing (25), the structure constant of the algebra and the dynamical variable of the tensor models can be identified as in (26). Therefore, it is possible to write down actions of the tensor models in terms of the algebra.

For simplicity, let me assume the total symmetry (27) of $C_{a b c}$, which requires the commutativity (28) of the algebra. It should be straightforward to extend the following discussions also to the general cases with noncommutativity. In the quadratic order of $C_{a b c}$, there exist two actions which are invariant under the orthogonal group symmetry (2), and they can be expressed with the algebraic language $\operatorname{as}^{38}$

$$
\begin{aligned}
& S_{1}^{(2)}=C_{a b c} C_{a b c}=\left\langle\phi_{a} \phi_{b} \mid \phi_{a} \phi_{b}\right\rangle, \\
& S_{2}^{(2)}=C_{a a c} C_{b b c}=\left\langle\phi_{a} \phi_{a} \mid \phi_{b} \phi_{b}\right\rangle .
\end{aligned}
$$

These equations can be checked by using $\phi_{a} \phi_{b}=C_{a b c} \phi_{c}$ under the identification (26).

More interesting forms appear in the quartic order. For example,

$$
\begin{aligned}
& S_{1}^{(4)}=C_{a b c} C_{a b d} C_{e f c} C_{e f d}=\left\langle\left(\phi_{a} \phi_{b}\right) \phi_{c} \mid\left(\phi_{a} \phi_{b}\right) \phi_{c}\right\rangle, \\
& S_{2}^{(4)}=C_{a b c} C_{a d e} C_{b d f} C_{c e f}=\left\langle\left(\phi_{a} \phi_{b}\right) \phi_{c} \mid \phi_{a}\left(\phi_{b} \phi_{c}\right)\right\rangle .
\end{aligned}
$$


A usage of this kind of algebraic expression is to find an action bounded from below to assure the stability of the tensor models. The action $S_{1}^{(4)}$ is positive definite, because it has a form of a norm square for the positive definite metric (25). On the other hand, $S_{2}^{(4)}$ is not so, but can be made it bounded from below by combining with $S_{1}^{(4)}$, as can be proven by

$$
S_{1}^{(4)}+S_{2}^{(4)}=\frac{1}{2}\left\langle\left(\phi_{a} \phi_{b}\right) \phi_{c}+\phi_{a}\left(\phi_{b} \phi_{c}\right) \mid\left(\phi_{a} \phi_{b}\right) \phi_{c}+\phi_{a}\left(\phi_{b} \phi_{c}\right)\right\rangle \geq 0
$$

It is obvious that one can construct various actions by considering such invariants of the algebra.

As described in Section III, the symmetry of such an action of a tensor model is given by the orthogonal group symmetry $O(N, R)$. On the other hand, as discussed in Section III, the 3-ary product (14) can be regarded as the generators of this symmetry under the gauge fixing condition (25) and the identification (26). Therefore the symmetry of the tensor models can be written algebraically by using the 3-ary transformation (18) as

$$
\delta_{a b} \phi_{c}=\left[\phi_{a}, \phi_{b} ; \phi_{c}\right]
$$

Since, on account of (15), the number of the independent choices of $\phi_{a}$ and $\phi_{b}$ agrees with the dimension of the orthogonal group $O(N, R)$, the 3-ary transformations (34) will span all the symmetry generators unless the configuration $C_{a b c}$ is fine-tuned not to be $\mathrm{so}^{39}$. In fact, by choosing appropriate elements $\phi_{a}$ and $\phi_{b}$, various transformations of physical interests can be constructed. In the following sections, Poincare transformations of the coordinates of fuzzy flat spacetimes will explicitly be given.

\section{THE ALGEBRA CORRESPONDING TO THE GAUSSIAN CONFIGURATIONS IN THE TENSOR MODELS}

In the study of emergent general relativity from the tensor models, a certain kind of configurations in the tensor models play important roles ${ }^{21}-26$. These configurations have Gaussian forms given by

$$
C_{p^{1} p^{2} p^{3}}=\exp \left(-\alpha\left(\left(p^{1}\right)^{2}+\left(p^{2}\right)^{2}+\left(p^{3}\right)^{2}\right)\right) \delta^{D}\left(p^{1}+p^{2}+p^{3}\right)
$$

where the indices are $D$-dimensional momentum, $p^{i}=\left(p_{1}^{i}, p_{2}^{i}, \ldots, p_{D}^{i}\right)$, and $\delta^{D}(\cdot)$ denotes the $D$-dimensional $\delta$-function. Here $(p)^{2}$ denotes the square of $D$-dimensional momentum 
Tensor models and 3-ary algebras

defined by

$$
(p)^{2}=g^{\mu \nu} p_{\mu} p_{\nu}
$$

where $g^{\mu \nu}$ is a constant real symmetric two-tensor. The parameter $\alpha$ is redundant in the sense that it can be absorbed into the redefinition of $g^{\mu \nu}$, but it is kept there for the dimensional reason. These Gaussian configurations satisfy the totally symmetric condition (27) in the previous section, and therefore define the class of fuzzy spaces discussed so far.

From the identification (26), the algebra corresponding to (35) is given by ${ }^{40}$,

$$
\phi_{p^{1}} \phi_{p^{2}}=C_{p^{1} p^{2} p^{3}} h^{p^{3} p^{4}} \phi_{p^{4}}=\exp \left(-2 \alpha\left(\left(p^{1}\right)^{2}+\left(p^{2}\right)^{2}+p^{1} \cdot p^{2}\right)\right) \phi_{p^{1}+p^{2}}
$$

where $p^{1} \cdot p^{2}$ denotes the inner product, $g^{\mu \nu} p_{\mu}^{1} p_{\nu}^{2}$. Because of the exponential factor in (37), the algebra (37) may be regarded as a deformation of the algebra of plane waves, $\phi_{p} \sim e^{i p x}$, in an ordinary spacetime, $\phi_{p^{1}} \phi_{p^{2}}=\phi_{p^{1}+p^{2}}$. The algebra (37) is commutative but nonassociative, and the parameter $\alpha$ characterizes the scale of the nonassociativity. The algebra is obviously invariant under the Poincare group in $D$-dimensions, and therefore it is physically interpreted as an algebra defining a $D$-dimensional nonassociative fuzzy flat spacetime $\stackrel{41}{ }$.

Now let me define the spacetime coordinates of this fuzzy spacetime. For the ordinary spacetime, the coordinates can be obtained by taking derivatives of the plane waves with respect to the momentum and putting the momentum to zero, $x^{\mu}=-\left.i \frac{\partial}{\partial p_{\mu}} e^{i p x}\right|_{p=0}$. Following this ordinary manner, let me define coordinates by

$$
\hat{x}^{\mu}=-\left.i \frac{\partial \phi_{p}}{\partial p_{\mu}}\right|_{p=0}=i \int d^{D} p \delta^{\mu}(p) \phi_{p}
$$

where

$$
\delta^{\mu}(p) \equiv \delta\left(p_{1}\right) \delta\left(p_{2}\right) \cdots \delta^{\prime}\left(p_{\mu}\right) \cdots \delta\left(p_{D}\right)
$$

Here $\delta^{\prime}(p)$ denotes the first derivative of the delta function defined by

$$
\int d p \delta^{\prime}(p) \phi_{p}=-\left.\frac{d \phi_{p}}{d p}\right|_{p=0}
$$

Similarly, for later convenience, let me generalize this to

$$
\left.\hat{x}^{\mu_{1} \mu_{2} \ldots \mu_{n}} \equiv(-i)^{n} \frac{\partial^{n} \phi_{p}}{\partial p_{\mu_{1}} \partial p_{\mu_{2}} \cdots \partial p_{\mu_{n}}}\right|_{p=0}=i^{n} \int d^{D} p \delta^{\mu_{1} \mu_{2} \ldots \mu_{n}}(p) \phi_{p}
$$


where $\delta^{\mu_{1} \mu_{2} \ldots \mu_{n}}(p)$ is defined similarly as (39). Then, using (37), the products of $\hat{x}^{\mu_{1} \mu_{2} \ldots \mu_{n}}$ are given by

$$
\hat{x}^{\mu_{1} \mu_{2} \ldots \mu_{n}} \hat{x}^{\nu_{1} \nu_{2} \ldots \nu_{m}}=i^{n+m} \int d^{D} q d^{D} q \delta^{\mu_{1} \mu_{2} \ldots \mu_{n}}(p) \delta^{\nu_{1} \nu_{2} \ldots \nu_{m}}(q) e^{-2 \alpha\left(p^{2}+q^{2}+p \cdot q\right)} \phi_{p+q} .
$$

From (42), it is easy to explicitly derive some low order products as

$$
\begin{aligned}
\phi_{0} \hat{x}^{\mu} & =\hat{x}^{\mu}, \\
\hat{x}^{\mu} \hat{x}^{\nu} & =2 \alpha g^{\mu \nu} \phi_{0}+\hat{x}^{\mu \nu}, \\
\hat{x}^{\mu \nu} \hat{x}^{\rho} & =4 \alpha g^{\mu \nu} \hat{x}^{\rho}+2 \alpha g^{\mu \rho} \hat{x}^{\nu}+2 \alpha g^{\nu \rho} \hat{x}^{\mu},
\end{aligned}
$$

where

$$
\phi_{0}=\left.\phi_{p}\right|_{p=0}
$$

Especially, from (43), (44) and (45), one obtains

$$
\begin{aligned}
{\left[\hat{x}^{\mu}, \hat{x}^{\nu} ; \hat{x}^{\rho}\right] } & =\left(\hat{x}^{\mu} \hat{x}^{\rho}\right) \hat{x}^{\nu}-\left(\hat{x}^{\nu} \hat{x}^{\rho}\right) \hat{x}^{\mu} \\
& =\left(2 \alpha g^{\mu \rho} \phi_{0}+\hat{x}^{\mu \rho}\right) \hat{x}^{\nu}-\left(2 \alpha g^{\nu \rho} \phi_{0}+\hat{x}^{\nu \rho}\right) \hat{x}^{\mu} \\
& =4 \alpha\left(g^{\mu \rho} \hat{x}^{\nu}-g^{\nu \rho} \hat{x}^{\mu}\right) .
\end{aligned}
$$

\section{THE 3-ARY PRODUCT OF COORDINATES AND SNYDER'S NONCOMMUTATIVE SPACETIME}

The 3-ary product defined in (14) does not satisfy the Leibnitz rule in general, but there exist the possibilities that subalgebras satisfy kinds of Leibnitz rules. In fact, one can explicitly check that the 3-ary product (47) of the coordinates satisfies the fundamental identity $\stackrel{42}{\underline{2}}$ (or Filippov identity $\stackrel{28}{22}$ ), which is given by

$$
\left[\hat{x}^{\mu}, \hat{x}^{\nu} ;\left[\hat{x}^{\rho}, \hat{x}^{\delta} ; \hat{x}^{\epsilon}\right]\right]=\left[\left[\hat{x}^{\mu}, \hat{x}^{\nu} ; \hat{x}^{\rho}\right], \hat{x}^{\delta} ; \hat{x}^{\epsilon}\right]+\left[\hat{x}^{\rho},\left[\hat{x}^{\mu}, \hat{x}^{\nu} ; \hat{x}^{\delta}\right] ; \hat{x}^{\epsilon}\right]+\left[\hat{x}^{\rho}, \hat{x}^{\delta} ;\left[\hat{x}^{\mu}, \hat{x}^{\nu} ; \hat{x}^{\epsilon}\right]\right]
$$

This kind of algebra is called a 3-Leibnitz algebra ${ }^{28,29}$.

The antisymmetry of the first two entries (15), the cyclic identity (30), and the fundamental identity (48) shows that the coordinates $\hat{x}^{\mu}$ and the 3 -ary product (14) form a Lie

triple system $27-29,43$. A Lie triple system is known to have an associated Lie algebra, and the same Lie triple system as the present case is explained in detail as an example in Ref. 43 (see 
also Refs. 27 29). The construction of a Lie algebra from the Lie triple system starts with formally defining some anti-symmetric commutators and new generators $\hat{M}^{\mu \nu}$ by relations,

$$
\begin{aligned}
4 \alpha \hat{M}^{\mu \nu} & \equiv\left[\hat{x}^{\mu}, \hat{x}^{\nu}\right]=-\left[\hat{x}^{\nu}, \hat{x}^{\mu}\right], \\
{\left[\hat{M}^{\mu \nu}, \hat{x}^{\rho}\right] } & =-\left[\hat{x}^{\rho}, \hat{M}^{\mu \nu}\right] \equiv \frac{1}{4 \alpha}\left[\hat{x}^{\mu}, \hat{x}^{\nu} ; \hat{x}^{\rho}\right]=g^{\mu \rho} \hat{x}^{\nu}-g^{\nu \rho} \hat{x}^{\mu},
\end{aligned}
$$

where the last equation is from (47). Here it is important to note that the commutator [, ] is only formally defined, and is not defined by products $[a, b]=a b-b a$, which identically vanishes on account of the commutativity of the algebra. The consistency of the formal definitions of the commutators as a Lie algebra is guaranteed by the antisymmetry of the first two entries (15), the cyclic identity (30), and the fundamental identity (48). Especially, from consistency, one obtaines

$$
\left[\hat{M}^{\mu \nu}, \hat{M}^{\rho \sigma}\right]=g^{\mu \rho} \hat{M}^{\nu \sigma}-g^{\mu \sigma} \hat{M}^{\nu \rho}-g^{\nu \rho} \hat{M}^{\mu \sigma}+g^{\nu \sigma} \hat{M}^{\mu \rho} .
$$

Therefore $\hat{M}^{\mu \nu}$ can be interpreted as the generators of the rotational (Lorentz) transformation of the coordinates $\hat{x}^{\mu}$ of a flat space(time) with an inverse metric $g^{\mu \nu}$.

The associated Lie algebra defined by (49), (50) and (51) agrees with that of the Snyder's noncommutative spacetime 445 . Derivation of the Snyder's noncommutative spacetime from a Lie triple system similarly as above has first been discussed in another kind of nonassociative spacetimes in Refs. 46 and 47. The two kinds of nonassociative spacetimes have different structures, but derive the same algebra of coordinates. This is probably because the coordinates defined in (38) are evaluated around $p=0$, and both of the nonassociative spacetimes have common infrared structures. These nonassociative spacetimes show interesting transmutation from nonassociativity to noncommutativity: the products of two $\hat{x}^{\mu}$ are commutative, but products of more than two coordinates show noncommutativity as in the case of the 3-ary product. Thus, 3-ary products and Lie triple systems open a new way of interpreting noncommutative spacetimes. As has been stressed in Refs. 46 and 47, this way of realizing noncommutative spacetimes can serve as a new solution to the problem of unwanted dimensions in noncommutative spacetimes. In Snyder's noncommutative spacetime, the algebra of the coordinates $\hat{x}^{\mu}$ does not close by themselves and $\hat{M}^{\mu \nu}$ appear as other coordinates. Therefore one has to confront a rather hard problem of making $\hat{M}^{\mu \nu}$ physically invisible. On the other hand, however, the $\hat{M}^{\mu \nu}$ introduced in (49) is just a label for representing a 3-ary operation, and does not represent any coordinates. 


\section{EXTENSION TO POINCARE SYMMETRY}

In the previous section, the rotational (Lorentz) transformation of the space(time) coordinates $\hat{x}^{\mu}$ has been shown to be realized by a 3-ary operation (50). In this section, it will be

shown that the translational transformation of the coordinates of the fuzzy flat spacetime can also be represented by a 3-ary operation.

What appears to be very strange in discussing the translational transformation of the spacetime coordinates $\hat{x}^{\mu}$ is that the 3 -ary algebra (47) of the coordinates $\hat{x}^{\mu}$ does not apparently seem invariant under the naive translation, $\hat{x}^{\mu} \rightarrow \hat{x}^{\mu}+v^{\mu}$, where $v^{\mu}$ is a $c$-number vector. In fact, the left-hand side of (47) does not change under the naive translation, while the last expression of (47) is shifted. On the other hand, however, the plane wave algebra (37) is obviously invariant under the phase rotations,

$$
\phi_{p} \rightarrow e^{i p \cdot v} \phi_{p}
$$

This is actually the same as the phase rotation generated by the translations in an ordinary spacetime. Therefore, to find the correct translational transformation on the fuzzy flat spacetime, one has to take good care of (52).

By the replacement (52), the coordinates defined in (38) will be transformed to

$$
\hat{x}^{\mu} \rightarrow-\left.i \frac{\partial e^{i p \cdot v} \phi_{p}}{\partial p_{\mu}}\right|_{p=0}=\hat{x}^{\mu}+v^{\mu} \phi_{0}
$$

where $\phi_{0}$ is defined in (46). Therefore the shift of the coordinates under the translation is actually given by $\delta \hat{x}^{\mu}=v^{\mu} \phi_{0}$, and not by a simple $c$-number vector. In fact, $\phi_{0}$ is not a trivial element, because, from (37),

$$
\phi_{0} \phi_{p}=e^{-2 \alpha(p)^{2}} \phi_{p}
$$

From (54) and (42), one also has

$$
\begin{aligned}
\phi_{0} \phi_{0} & =\phi_{0}, \\
\phi_{0} \hat{x}^{\mu \nu} & =4 \alpha g^{\mu \nu} \phi_{0}+\hat{x}^{\mu \nu} .
\end{aligned}
$$


Then the shift of the left-hand side of (47) by (53) can be computed as

$$
\begin{aligned}
\delta\left[\hat{x}^{\mu}, \hat{x}^{\nu} ; \hat{x}^{\rho}\right]= & \delta\left(\left(\hat{x}^{\mu} \hat{x}^{\rho}\right) \hat{x}^{\nu}-\left(\hat{x}^{\nu} \hat{x}^{\rho}\right) \hat{x}^{\mu}\right) \\
= & \left(v^{\mu} \phi_{0} \hat{x}^{\rho}\right) \hat{x}^{\nu}+\left(\hat{x}^{\mu} v^{\rho} \phi_{0}\right) \hat{x}^{\nu}+\left(\hat{x}^{\mu} \hat{x}^{\rho}\right) v^{\nu} \phi_{0} \\
& \quad-\left(v^{\nu} \phi_{0} \hat{x}^{\rho}\right) \hat{x}^{\mu}-\left(\hat{x}^{\nu} v^{\rho} \phi_{0}\right) \hat{x}^{\mu}-\left(\hat{x}^{\nu} \hat{x}^{\rho}\right) v^{\mu} \phi_{0} \\
= & 4 \alpha g^{\mu \rho} v^{\nu} \phi_{0}-4 \alpha g^{\nu \rho} v^{\mu} \phi_{0},
\end{aligned}
$$

where I have used (43), (44), (55) and (56). The last expression indeed agrees with the shift of the last expression of (47), and therefore (47) is invariant under the translational symmetry (53) on the fuzzy flat spacetime.

Thus, to properly incorporate the translational symmetry of the fuzzy flat spacetime, the 3 -ary algebra of the coordinates $\hat{x}^{\mu}$ should be extended to include the element $\phi_{0}$. From (43), (44), (55) and (하), one can explicitly obtain

$$
\begin{aligned}
{\left[\hat{x}^{\mu}, \phi_{0} ; \hat{x}^{\nu}\right]=-\left[\phi_{0}, \hat{x}^{\mu} ; \hat{x}^{\nu}\right] } & =4 \alpha g^{\mu \nu} \phi_{0}, \\
{\left[\text { The others with } \phi_{0}\right] } & =0,
\end{aligned}
$$

and the extended 3-ary algebra is closed. Then one finds that the translational transformation (53) can be represented by

$$
\delta \hat{x}^{\mu}=\left[v_{\nu} \hat{x}^{\nu}, \phi_{0} ; \hat{x}^{\mu}\right] .
$$

In fact, the 3-ary algebra extended with $\phi_{0}$ can be regarded as the 3-ary algebra of the kind (47) extended with a new coordinate $\phi_{0}$ with a degenerate metric,

$$
g^{\mu 0}=g^{0 \mu}=g^{00}=0 .
$$

This can be checked by comparing (47) with (58). Therefore, the extended algebra also forms a Lie triple system. Following the same procedure as in the previous section, the associated Lie algebra can be shown to contain the Poincare Lie algebra,

$$
\begin{aligned}
{\left[\hat{T}^{\mu}, \hat{T}^{\nu}\right] } & =0, \\
{\left[\hat{M}^{\mu \nu}, \hat{T}^{\rho}\right] } & =g^{\mu \rho} \hat{T}^{\nu}-g^{\nu \rho} \hat{T}^{\mu}, \\
{\left[\hat{M}^{\mu \nu}, \hat{M}^{\rho \sigma}\right] } & =g^{\mu \rho} \hat{M}^{\nu \sigma}-g^{\mu \sigma} \hat{M}^{\nu \rho}-g^{\nu \rho} \hat{M}^{\mu \sigma}+g^{\nu \sigma} \hat{M}^{\mu \rho},
\end{aligned}
$$

where

$$
\begin{aligned}
4 \alpha \hat{T}^{\mu} & \equiv\left[\hat{x}^{\mu}, \phi_{0}\right]=-\left[\phi_{0}, \hat{x}^{\mu}\right], \\
{\left[\hat{T}^{\mu}, \hat{x}^{\nu}\right] } & =-\left[\hat{x}^{\nu}, \hat{T}^{\mu}\right] \equiv \frac{1}{4 \alpha}\left[\hat{x}^{\mu}, \phi_{0} ; \hat{x}^{\nu}\right]=g^{\mu \nu} \phi_{0} .
\end{aligned}
$$




\section{SUMMARY, DISCUSSIONS AND FUTURE PROSPECTS}

In this paper, I have studied the idea, first presented in Ref. 18, that the rank-three tensor models can be interpreted as theory of dynamical fuzzy spaces. The algebra of functions on a corresponding fuzzy space is shown to be constrained to have a certain cyclic property, which comes from the generalized hermiticity condition on the three-index tensor in the rank-three tensor models. The actions of the rank-three tensor models can be represented algebraically, and such algebraic description will provide a new tool to study the rank-three tensor models.

The most important implication of wide interest of this paper would be that the cyclic property of algebras may be used to define an interesting class of fuzzy spaces. As discussed in Section III, the cyclic property is the essential feature to guarantee the 3-ary operation to be a transformation which conserves the metric of the algebra. Such metric conserving transformations are of physical importance, since they should describe various physically meaningful unitary transformations on fuzzy spaces including the ones corresponding to the diffeomorphisms in usual spacetimes ${ }^{24}$. It is not generally possible to obtain such metric conserving transformations in terms of products of functions as in the 3-ary operation of this paper, if the algebra of functions does not satisfy the cyclic property. Moreover, the cyclic property makes it possible to systematically generate metric conserving transformations in terms of $n$-ary $\underline{\underline{48}}$ and supersymmetric ${ }^{49}$ operations. It should be interesting to systematically study various aspects of spacetime symmetry transformations on physically motivated fuzzy spacetimes from this new perspective. It would also be possible that the algebraic structure presented in this paper may have some applications to the description of the nonassociative fuzzy spacetimes discussed in D-brane $\operatorname{setups}^{50}-52$.

While commutation relations of coordinates can well describe some symmetric noncommutative spacetimes such as the noncommutative two-sphere ${ }^{53}$, n-ary algebras of coordinates can be expected to characterize symmetric fuzzy spaces with nonassociativity $\underline{\underline{50}} \underline{\underline{52}}$. To pursue this expectation further, the commutative nonassociative fuzzy flat spacetimes motivated from the tensor models have been investigated, and a 3-ary relation of coordinates has been found for the fuzzy flat spacetimes. While the Lorentz symmetry is obviously satisfied in the obtained 3-ary relation, it apparently contradicts with the translational symmetry of the fuzzy flat spacetimes. But a detailed analysis of the flat spacetime algebra has shown that 
the 3-ary relation is indeed consistent with a 3-ary translational transformation. This aspect is in good parallel with the fact that the apparently violated translational symmetry of a three-dimensional noncommutative space obtained from lattice gravity is indeed conserved by a Hopf algebraic translation $\underline{54} \underline{-56}$.

The 3-ary relation of the coordinates of the fuzzy flat spacetimes has been shown to form a Lie triple system, and the associated Lie algebra has been shown to be identical with the Snyder's noncommutative spacetime. A similar result has been obtained in another kind of nonassociative spacetimes in Refs. 46 and 47 . These examples show interesting transmutation from nonassociativity to noncommutativity, and, as stressed in Refs. 46 and 47, can provide a solution to the problem of unwanted dimensions in noncommutative spacetimes. It would be interesting to study whether other noncommutative spacetimes can also be embedded into nonassociative ones in similar manners.

What seems especially interesting is the appearance of the 3-ary product, by which the symmetry of the rank-three tensor models can be represented. This new way of describing symmetries by 3-ary algebras seems to contain a conceptual interest in origins of symmetries. In a usual framework, a symmetry is a given input rather than an emergent phenomenon. On the other hand, however, the form of the symmetry transformation by the 3-ary algebra in the tensor models suggests that the symmetry can only appear when a background is generated. This new aspect of symmetry should be explored further in the context of the general idea that spacetime and gravity are emergent phenomena ${ }^{57} \underline{68}$.

\section{ACKNOWLEDGMENTS}

Part of the contents of this paper has first been presented in the lecture at "Shinshu Winter School", held March 10-12, 2011, in Shiga Heights Villa, Ochanomizu University. The author would like to thank the organizers of this winter school for giving him the opportunity to get the main ideas of this paper on this occasion. The author would also like to thank M. Axenides, E. Floratos, F. Girelli, and S. Ramgoolam for e-mail correspondence. 


\section{REFERENCES}

${ }^{1}$ Jan Ambjorn, Bergfinnur Durhuus, and Thordur Jonsson, "Three-dimensional simplicial quantum gravity and generalized matrix models," Mod.Phys.Lett. A6, 1133-1146 (1991).

${ }^{2}$ Naoki Sasakura, "Tensor model for gravity and orientability of manifold," Mod.Phys.Lett. A6, 2613-2624 (1991).

${ }^{3}$ Neil Godfrey and Mark Gross, "Simplicial quantum gravity in more than two-dimensions," Phys.Rev. D43, 1749-1753 (1991).

${ }^{4}$ D.V. Boulatov, "A Model of three-dimensional lattice gravity," Mod.Phys.Lett. A7, 16291646 (1992), arXiv:hep-th/9202074 [hep-th].

${ }^{5}$ Hirosi Ooguri, "Topological lattice models in four-dimensions," Mod.Phys.Lett. A7, 27992810 (1992), arXiv:hep-th/9205090 [hep-th].

${ }^{6}$ Roberto De Pietri, Laurent Freidel, Kirill Krasnov, and Carlo Rovelli, "Barrett-Crane model from a Boulatov-Ooguri field theory over a homogeneous space," Nucl.Phys. B574, 785-806 (2000), arXiv:hep-th/9907154 [hep-th].

${ }^{7}$ This kind of models are also called group field theory.

${ }^{8}$ Roberto De Pietri and Carlo Petronio, "Feynman diagrams of generalized matrix models and the associated manifolds in dimension 4," J.Math.Phys. 41, 6671-6688 (2000), arXiv:gr-qc/0004045 [gr-qc].

${ }^{9}$ Razvan Gurau, "The complete $1 / \mathrm{N}$ expansion of colored tensor models in arbitrary dimension," (2011), arXiv:1102.5759 [gr-qc]].

${ }^{10}$ Razvan Gurau, "The 1/N expansion of colored tensor models," Annales Henri Poincare 12, 829-847 (2011), arXiv:1011.2726 [gr-qc].

${ }^{11}$ Joseph Ben Geloun, Razvan Gurau, and Vincent Rivasseau, "EPRL/FK Group Field Theory," Europhys.Lett. 92, 60008 (2010), arXiv:1008.0354 [hep-th].

${ }^{12}$ Razvan Gurau, "Lost in Translation: Topological Singularities in Group Field Theory," Class.Quant.Grav. 27, 235023 (2010), arXiv:1006.0714 [hep-th].

${ }^{13}$ Razvan Gurau, "Topological Graph Polynomials in Colored Group Field Theory," Annales Henri Poincare 11, 565-584 (2010), arXiv:0911.1945 [hep-th].

${ }^{14}$ Razvan Gurau, "Colored Group Field Theory," Commun.Math.Phys. 304, 69-93 (2011), arXiv:0907.2582 [hep-th].

${ }^{15}$ Aristide Baratin, Florian Girelli, and Daniele Oriti, "Diffeomorphisms in group field the- 
ories," Phys.Rev. D83, 104051 (2011), arXiv:1101.0590 [hep-th].

${ }^{16}$ J. Ambjorn, J. Jurkiewicz, and R. Loll, "Causal Dynamical Triangulations and the Quest for Quantum Gravity," (2010), arXiv:1004.0352 [hep-th].

${ }^{17}$ O. Lauscher and M. Reuter, "Fractal spacetime structure in asymptotically safe gravity," JHEP 0510, 050 (2005), arXiv:hep-th/0508202 [hep-th].

${ }^{18}$ Naoki Sasakura, "An Invariant approach to dynamical fuzzy spaces with a three-index variable," Mod.Phys.Lett. A21, 1017-1028 (2006), arXiv:hep-th/0506192 [hep-th].

${ }^{19}$ Naoki Sasakura, "An Invariant approach to dynamical fuzzy spaces with a three-index variable: Euclidean models," (2005), arXiv:hep-th/0511154 [hep-th].

${ }^{20}$ Naoki Sasakura, "Tensor model and dynamical generation of commutative nonassociative fuzzy spaces," Class.Quant.Grav. 23, 5397-5416 (2006), arXiv:hep-th/0606066 [hep-th].

${ }^{21}$ Naoki Sasakura, "The Fluctuation spectra around a Gaussian classical solution of a tensor model and the general relativity," Int.J.Mod.Phys. A23, 693-718 (2008), arXiv:0706.1618 [hep-th].

${ }^{22}$ Naoki Sasakura, "The Lowest modes around Gaussian solutions of tensor models and the general relativity," Int.J.Mod.Phys. A23, 3863-3890 (2008), arXiv:0710.0696 [hep-th].

${ }^{23}$ Naoki Sasakura, "Emergent general relativity on fuzzy spaces from tensor models," Prog.Theor.Phys. 119, 1029-1040 (2008), arXiv:0803.1717 [gr-qc].

${ }^{24}$ Naoki Sasakura, "Gauge fixing in the tensor model and emergence of local gauge symmetries," Prog.Theor.Phys. 122, 309-322 (2009), arXiv:0904.0046 [hep-th].

${ }^{25}$ Naoki Sasakura, "Emergent general relativity in the tensor models possessing Gaussian classical solutions," AIP Conf.Proc. 1243, 76-86 (2010), arXiv:0911.1170 [hep-th].

${ }^{26}$ Naoki Sasakura, "A Renormalization procedure for tensor models and scalar-tensor theories of gravity," Int.J.Mod.Phys. A25, 4475-4492 (2010), arXiv:1005.3088 [hep-th].

${ }^{27}$ Jose Miguel Figueroa-O'Farrill, "Three lectures on 3-algebras," (2008), arXiv:0812.2865 [hep-th].

${ }^{28}$ Jose A. de Azcarraga and Jose M. Izquierdo, "n-ary algebras: A Review with applications," J.Phys.A A43, 293001 (2010), arXiv:1005.1028 [math-ph].

${ }^{29}$ J.A. de Azcarraga and J.M. Izquierdo, "Topics on n-ary algebras," J.Phys.Conf.Ser. 284, 012019 (2011), arXiv:1102.4194 [math-ph].

${ }^{30}$ Yoichiro Nambu, "Generalized Hamiltonian dynamics," Phys.Rev. D7, 2405-2414 (1973).

${ }^{31}$ Jonathan Bagger and Neil Lambert, "Modeling Multiple M2's," Phys.Rev. D75, 045020 
(2007), arXiv:hep-th/0611108 [hep-th].

${ }^{32}$ Andreas Gustavsson, "Algebraic structures on parallel M2-branes," Nucl.Phys. B811, 6676 (2009), arXiv:0709.1260 [hep-th]

${ }^{33}$ Jonathan Bagger and Neil Lambert, "Gauge symmetry and supersymmetry of multiple M2-branes," Phys.Rev. D77, 065008 (2008), arXiv:0711.0955 [hep-th].

${ }^{34}$ In this paper, an algebra is allowed to be nonassociative in general. This is physically required to circumvent the Wedderburn's theorem, which roughly requires a finite associative algebra to be a matrix algebra. A matrix algebra would be too simple to represent a fuzzy space with geometric properties.

${ }^{35}$ Because of the possible nonassociativity of the algebra, the order of the products must be explicitly indicated.

${ }^{36}$ However, for example, see Ref. 69 and the references therein for some efforts to realize the Leibnitz rule in quantization of Nambu bracket.

${ }^{37}$ R. D. Schafer, An introduction to nonassociative algebras (Academic Press, 1966).

${ }^{38}$ The repeated indices are assumed to be summed over.

${ }^{39}$ More detailed discussions will be given elsewhere ${ }^{48}$.

${ }^{40}$ The metric in the coordinate basis is given by $h_{x_{1} x_{2}}=\delta^{D}\left(x_{1}-x_{2}\right)$, which has the form of the gauge-fixing condition (25). Then, by taking Fourier transformation of $h_{x_{1} x_{2}}$, the metric in the plane wave basis is given by $h_{p^{1} p^{2}}=\delta^{D}\left(p^{1}+p^{2}\right)$.

${ }^{41}$ Yuya Sasai and Naoki Sasakura, "One-loop unitarity of scalar field theories on Poincare invariant commutative nonassociative spacetimes," JHEP 0609, 046 (2006), arXiv:hep-th/0604194 [hep-th].

${ }^{42}$ Leon Takhtajan, "On Foundation of the generalized Nambu mechanics (second version)," Commun.Math.Phys. 160, 295-316 (1994), arXiv:hep-th/9301111 [hep-th].

${ }^{43} \mathrm{~S}$. Okubo, "Construction of Lie superalgebras from triple product systems," AIP Conf.Proc. 687, 33-40 (2003).

${ }^{44}$ Hartland S. Snyder, "Quantized space-time," Phys.Rev. 71, 38-41 (1947).

${ }^{45}$ The parameter $4 \alpha$ corresponds to $a^{2}$ in Ref. 44 .

${ }^{46}$ Florian Girelli, "Snyder Space-Time: K-Loop and Lie Triple System," SIGMA 6, 074 (2010), arXiv:1009.4762 [math-ph].

${ }^{47}$ Florian Girelli and Etera R. Livine, "Scalar field theory in Snyder space-time: Alternatives," JHEP 1103, 132 (2011), arXiv:1004.0621 [hep-th]. 
${ }^{48}$ Naoki Sasakura, "Tensor models and hierarchy of n-ary algebras," Int.J.Mod.Phys. A26, 3249-3258 (2011), arXiv:1104.5312 [hep-th].

${ }^{49}$ Naoki Sasakura, "Super tensor models, super fuzzy spaces and super n-ary transformations," Int.J.Mod.Phys. A26, 4203-4216 (2011), arXiv:1106.0379 [hep-th].

${ }^{50}$ Paul de Medeiros and Sanjaye Ramgoolam, "Non-associative gauge theory and higher spin interactions," JHEP 0503, 072 (2005), arXiv:hep-th/0412027 [hep-th].

${ }^{51}$ Sanjaye Ramgoolam, "Towards gauge theory for a class of commutative and nonassociative fuzzy spaces," JHEP 0403, 034 (2004), arXiv:hep-th/0310153 [hep-th].

${ }^{52}$ Sanjaye Ramgoolam, "On spherical harmonics for fuzzy spheres in diverse dimensions," Nucl.Phys. B610, 461-488 (2001), arXiv:hep-th/0105006 [hep-th].

${ }^{53}$ J. Madore, "The Fuzzy sphere," Class.Quant.Grav. 9, 69-88 (1992).

${ }^{54}$ Laurent Freidel and Etera R. Livine, "Effective 3-D quantum gravity and non-commutative quantum field theory," Phys.Rev.Lett. 96, 221301 (2006), arXiv:hep-th/0512113 [hep-th].

${ }^{55}$ Laurent Freidel and Etera R. Livine, "Ponzano-Regge model revisited III: Feynman diagrams and effective field theory," Class.Quant.Grav. 23, 2021-2062 (2006), arXiv:hep-th/0502106 [hep-th].

${ }^{56}$ Yuya Sasai and Naoki Sasakura, "Braided quantum field theories and their symmetries," Prog.Theor.Phys. 118, 785-814 (2007), arXiv:0704.0822 [hep-th],

${ }^{57}$ Erik P. Verlinde, "On the Origin of Gravity and the Laws of Newton," JHEP 1104, 029 (2011), arXiv:1001.0785 [hep-th].

${ }^{58}$ T. Padmanabhan, "Gravity as an emergent phenomenon," Int.J.Mod.Phys. D17, 591-596 (2008).

${ }^{59}$ David Berenstein, "Large N BPS states and emergent quantum gravity," JHEP 0601, 125 (2006), arXiv:hep-th/0507203 [hep-th].

${ }^{60}$ Gary T. Horowitz and Joseph Polchinski, "Gauge/gravity duality," (2006), in 'Towards Quantum Gravity'. Edited by Daniele Oriti. Cambridge University Press, arXiv:gr-qc/0602037 [gr-qc].

${ }^{61}$ Hai Lin, Oleg Lunin, and Juan Martin Maldacena, "Bubbling AdS space and 1/2 BPS geometries," JHEP 0410, 025 (2004), arXiv:hep-th/0409174 [hep-th].

${ }^{62}$ Jungjai Lee and Hyun Seok Yang, "Quantum Gravity from Noncommutative Spacetime," arXiv:1004.0745 [hep-th].

${ }^{63}$ Harold Steinacker, "Emergent Geometry and Gravity from Matrix Models: an Introduc- 
Tensor models and 3-ary algebras

tion," Class.Quant.Grav. 27, 133001 (2010), arXiv:1003.4134 [hep-th].

${ }^{64}$ David E. Berenstein, Masanori Hanada, and Sean A. Hartnoll, "Multi-matrix models and emergent geometry," JHEP 0902, 010 (2009), arXiv:0805.4658 [hep-th].

${ }^{65}$ Johanna Erdmenger, Rene Meyer, and Jeong-Hyuck Park, "Spacetime Emergence in the Robertson-Walker Universe from a Matrix model," Phys.Rev.Lett. 98, 261301 (2007), arXiv:0705.1586 [hep-th].

${ }^{66}$ Nathan Seiberg, "Emergent spacetime," arXiv:hep-th/0601234 [hep-th].

${ }^{67}$ H. Kawai, Shoichi Kawamoto, Tsunehide Kuroki, T. Matsuo, and S. Shinohara, "Mean field approximation of IIB matrix model and emergence of four-dimensional space-time," Nucl.Phys. B647, 153-189 (2002), arXiv:hep-th/0204240 [hep-th].

${ }^{68}$ Daniele Oriti, "A Quantum field theory of simplicial geometry and the emergence of spacetime," J.Phys.Conf.Ser. 67, 012052 (2007), arXiv:hep-th/0612301 [hep-th].

${ }^{69}$ Minos Axenides and Emmanuel Floratos, "Nambu-Lie 3-Algebras on Fuzzy 3-Manifolds," JHEP 0902, 039 (2009), arXiv:0809.3493 [hep-th]. 